\title{
Gibbs measures for partially hyperbolic attractors
}

\author{
YA. B. PESIN AND YA. G. SINAI \\ L. D. Landau Institute for Theoretical Physics, Academy of Sciences of the USSR, \\ 142432 Chernogolovka, Moscow Region, USSR
}

(Received 20 January 1982)

Abstract. We consider iterates of absolutely continuous measures concentrated in a neighbourhood of a partially hyperbolic attractor. It is shown that limit points can be measures which have conditional measures of a special form for any partition into subsets of unstable manifolds.

\section{Introduction: Formulation of main results}

1.1. Let $M$ be a smooth Riemannian manifold, $U \subset M$ be an open set, $f: U \rightarrow M$ be a $C^{1+\alpha}$-diffeomorphism of $M$. A subset $\Lambda$ in $M$ is called a partially hyperbolic attractor if it satisfies the following conditions:

(A1) $\Lambda$ is closed and $f$-invariant;

(A2) the $\operatorname{map} f \mid \Lambda$ is partially hyperbolic;

(A3) there exists a neighbourhood $U_{0} \subset U$ of the set $\Lambda$ such that

$$
\overline{f\left(U_{0}\right)} \subset U_{0} \text { and } \Lambda=\bigcap_{n \geq 0} f^{n}\left(U_{0}\right) .
$$

Let us denote by $\Gamma^{0}(T \Lambda)$ the Banach space of continuous vector fields on $\Lambda$ with the uniform norm. The diffeomorphism $f$ induces a linear operator $f_{*}$ in $\Gamma^{0}(T \Lambda)$, given by the formula

$$
\left(f_{*} v\right)(x)=d f v\left(f^{-1}(x)\right), \quad x \in \Lambda, v \in \Gamma^{0}(T \Lambda) .
$$

It is known (cf. $[11,12]$ ), that the spectrum of the complexification of $f_{*}$ consists of whole circles. The condition (A2) means (cf. [3]) that the spectrum is contained in two rings whose radii are $\lambda_{1}^{\prime}, \mu_{1}^{\prime}, \lambda_{2}^{\prime}, \mu_{2}^{\prime}$ respectively and

$$
\infty>\lambda_{1}^{\prime} \geq \mu_{1}^{\prime}>\lambda_{2}^{\prime} \geq \mu_{2}^{\prime}>0, \mu_{1}^{\prime}>1 \text {. }
$$

There are continuous sub-bundles of the tangent bundle $T \Lambda$ invariant with respect to $d f$ which correspond to the components of the spectrum, lying in these rings. We denote them by $E^{u}$ and $E^{s 0}$ respectively. These distributions have the following properties, with respect to a special (so-called Lyapunov) Riemannian metric:

(1) $E^{u}(x) \oplus E^{s 0}(x)=T_{x} M$ for any $x \in \Lambda$;

(2) there exist numbers $\lambda_{1}, \mu_{1}, \lambda_{2}, \mu_{2}$ such that

$$
\infty>\lambda_{1}>\lambda_{1}^{\prime} \geqslant \mu_{1}^{\prime}>\mu_{1}>\lambda_{2}>\lambda_{2}^{\prime} \geq \mu_{2}^{\prime}>\mu_{2}>0, \quad \mu_{1}>1
$$


and for any $x \in \Lambda, n \geq 0$

$$
\begin{gathered}
\lambda_{1}^{n} \geq\left\|d f^{n} \mid E^{u}(x)\right\| \geq \mu_{1}^{n} \\
\lambda_{2}^{n} \geq\left\|d f^{n} \mid E^{s 0}(x)\right\| \geq \mu_{2}^{n} .
\end{gathered}
$$

We assume that the subspaces $E^{\prime \prime}(x)$ have the same dimension for any $x \in \Lambda$.

It is known (cf., for example, [12]) that for any $x \in \Lambda$ there exists a smooth submanifold $W_{\text {loc }}^{u}(x)$ having the following characteristic property: for any $y \in$ $W_{\text {loc }}^{u}(x)$ and $n \geq 0$

$$
\rho\left(f^{-n}(x), f^{-n}(y)\right) \leq C \mu_{1}^{-n} \rho(x, y)
$$

(here $C>0$ is a constant, independent on $x, y$ and $n$ and $\rho$ is the induced distance function on $M)$. Moreover $T_{x} W_{\text {loc }}^{u}(x)=E^{u}(x)$. The submanifold $W_{\text {loc }}^{u}(x)$ is called a local unstable manifold at the point $x \in \Lambda$. The global unstable manifold at $x \in \Lambda$ is defined by the expression

$$
W^{u}(x)=\bigcup_{-\infty<n<\infty} f^{n}\left(W_{\text {loc }}^{u}\left(f^{-n}(x)\right) .\right.
$$

Proposition 1. (1) For any neighbourhood $V_{0} \subset U_{0}$ of $\Lambda$

$$
\Lambda=\bigcap_{n \geq 0} f^{n}\left(V_{0}\right)
$$

(2) The set $\Lambda$ is locally maximal (i.e. for any compact f-invariant set $\Lambda \subset U_{0}$ we have $\left.\Lambda^{\prime} \subset \Lambda\right)$.

(3) $W^{u}(x) \subset \Lambda$ for any $x \in \Lambda$; moreover the sets $W^{u}(x), x \in \Lambda$ form an f-invariant $C^{1}$-foliation on $\Lambda$ (see the corresponding definition in [14]).

(4) There exists $C>0$ such that for any $x \in \Lambda, y \in W^{u}(x)$ and any $n \geq 0$

$$
\rho^{u}\left(f^{-n}(x), f^{-n}(y)\right) \leq C\left(\mu_{1}\right)^{-n} \rho^{u}(x, y),
$$

where $\rho^{u}$ is the distance on $W^{u}(x)$ induced by the Riemannian metric.

1.2. Let us denote by $\mathscr{F}(\Lambda)$ the set of all non-vanishing continuous functions $\phi$ on $\Lambda$ satisfying the Hölder condition, i.e.

$$
|\phi(x)-\phi(y)| \leq C \rho(x, y)^{\alpha} \text { for any } x, y \in \Lambda
$$

and some $C>0, \alpha \in(0,1)$ independent on $x, y \in \Lambda$. For any $x \in \Lambda, y \in W^{u}(x)$ and any integer $n>0$ we define the function

$$
\rho_{\phi, n}(x, y)=\prod_{K=0}^{n-1}\left[\phi\left(f^{-K}(x)\right)\right]\left[\phi\left(f^{-K}(y)\right)\right]^{-1} .
$$

Let us denote by $B^{u}(x, r)$ the open ball on the submanifold $W^{u}(x)$ with centre at $x$ and radius $r$. The properties of $\rho_{\phi, n}$, stated in the following proposition, have already been obtained before essentially in $[1,2]$.

Proposition 2. (1) For any $x \in \Lambda, y \in W^{u}(x)$ there exists the limit

$$
\rho_{\phi}(x, y)=\lim _{n \rightarrow \infty} \rho_{\phi, n}(x, y)>0 \text {. }
$$


Moreover, for any $\varepsilon>0, r>0$, there exists $N=N(\varepsilon, r)$ such that for every $n \geq N$

$$
\max _{x \in \Lambda} \max _{y \in \bar{B}^{u}(x, r)}\left|\rho_{\phi, n}(x, y)-\rho_{\phi}(x, y)\right| \leq \varepsilon .
$$

(2) The function $\rho_{\phi}(x, y)$ is continuous; more exactly for any $x \in \Lambda, y \in W^{u}(x)$ and any two sequences $\left\{x_{n}\right\},\left\{y_{n}\right\}$ such that $x_{n} \rightarrow x, y_{n} \rightarrow y, x_{n} \in \Lambda, y_{n} \in W^{u}\left(x_{n}\right)$ and value $\rho^{\mu}\left(x_{n}, y_{n}\right)$ is bounded from above uniformly on $n$ we have

$$
\lim _{n \rightarrow \infty} \rho_{\phi}\left(x_{n}, y_{n}\right)=\rho_{\phi}(x, y)
$$

(3) For any $x \in \Lambda, y, z \in W^{u}(x)$

$$
\rho_{\phi}(x, y) \rho_{\phi}(y, z)=\rho_{\phi}(x, z) .
$$

1.3. Let $x \in \Lambda$ and $W$ be a local manifold passing through $x$ transversally to foliation $W^{u}\left(\operatorname{dim} W=\operatorname{dim} E^{s 0}(x)\right)$. We call a $\Lambda$-rectangle at $x$ the set

$$
\Pi=\Pi(x, W, r)=\bigcup_{y \in W \cap \Lambda} B^{u}(y, r) .
$$

Let us assume that $r$ is small and denote by $\nu^{u}$ the measure on $W^{u}(y)$, induced by the Riemannian metric and by $\xi=\xi(\Pi)$ the partition of $\Pi(x, W, r)$ onto the sets $B^{u}(y, r)$. This partition being continuous it is measurable with respect to any Borel measure on $\Lambda$. We denote by $\chi(y), y \in \Pi(x, W, r)$ the point in $W \cap \Lambda$ such that $y \in B^{u}(\chi(y), r)$. (It is evident that such a point is uniquely defined.)

Let $\mu$ be a Borel measure on $\Lambda$. Let us fix a function $\phi \in \mathscr{F}(\Lambda)$, a point $x \in \Lambda$ and a $\Lambda$-rectangle $\Pi(x, W, r)$ and denote by $\mu_{\xi}(y)$ the conditional measures on the elements $C_{\xi}(y)=B^{u}(\chi(y), r)$ of the partition $\xi(\Pi)$. We shall say that the measure $\mu$ is Gibbs $(u, \phi)$-measure if for any $x \in \Lambda$ and any $\Lambda$-rectangle $\Pi(x, W, r)$

$$
d \mu_{\xi}(y)=r(\chi(y)) \rho_{\varphi}(\chi(y), y) d \nu^{u}(y),
$$

where the 'normalizing factor' (partition function $r(\chi(y)$ ) is given by the equality

$$
r(\chi(y))=\left[\int_{B^{u}\left(x(y)_{r}\right)} \rho_{\phi}(\chi(y), y) d \nu^{u}(y)\right]^{-1} .
$$

1.4. Let $\Phi(\Lambda)$ be the set of all normalized $f$-invariant Gibbs $(u, \phi)$-measures on $\Lambda$, $\phi \in \mathscr{F}(\Lambda)$. For $\mu \in \Phi(\Lambda)$ and $x \in \Lambda$ we denote by $\mathscr{J}_{\mu}^{u}(x)$ the Jacobian of the map $d f \mid E^{u}(x)$ with respect to $\mu$.

THEOREM 1. Let $\mu \in \Phi(\Lambda)$. The entropy $h_{\mu}(f \mid \Lambda)$ of $f \mid \Lambda$ with respect to $\mu$ satisfies the inequality

$$
h_{\mu}(f \mid \Lambda) \geq \int_{\Lambda} \ln \mathscr{J}_{\mu}^{u}(x) d \mu(x)>0
$$

The following statement plays an important role in the study of ergodic properties of the automorphism $f \mid \Lambda$.

THEOREM 2. There exist a set $\Lambda^{\prime} \subset \Lambda$ and a measurable partition $\xi$ of $\Lambda^{\prime}$ such that

(1) $\Lambda^{\prime}$ is open in $\Lambda$ and f-invariant; 
(2) for any $x \in \Lambda^{\prime}$ the element $C_{\xi}(x)$ is an open connected subset of the layer $W^{u}(x)$; if $x$ is forward recurrent then $\operatorname{diam} C_{\xi}(x) \leq \mathrm{const}$ (independent on $\left.x\right) ; \nu^{\mu}\left(\partial C_{\xi}(x)\right)=0$;

(3) $f^{-1} \xi \geq \xi$;

(4) $\bigvee_{n \geq 0} f^{-n} \xi=\varepsilon$;

(5) if $\mu \in \Phi(\Lambda)$ is an ergodic measure then

$$
\mu\left(\Lambda^{\prime}\right)=1 \text { and } \bigwedge_{n \geq 0} f^{n} \xi=\nu\left(W^{u}\right)
$$

where $\nu\left(W^{u}\right)$ is the measurable hull of the partition of $\Lambda$ into the layers of the foliation $W^{u}$ with respect to $\mu$.

Analogous statements for other cases can be found in $[6,8,10]$.

1.5. We denote by $\mathscr{J}^{u}(x), x \in \Lambda$ the Jacobian of the map $d f \mid E^{u}(x)$ with respect to the Riemannian volume $\nu$. As the distribution $E^{u}$ satisfies Hölder's condition (cf. [3]) $\mathscr{J}^{u} \in \mathscr{F}(\Lambda)$. We shall call a Gibbs $\left(u, \mathscr{J}^{u}\right)$-measure simply a Gibbs $u$-measure.

Let us fix a neighbourhood $V_{0} \subset U_{0}$ of $\Lambda$ and consider a measure in $V_{0}$ having a non-negative density $\Psi(x)$ with respect to Riemannian volume $\nu$ such that

$$
\operatorname{supp} \Psi(x) \subset \bar{V}_{0}, \quad \int_{V_{0}} \Psi(x) d \nu(x)=1 .
$$

Let us denote by $C\left(V_{0}\right)$ the Banach space of all continuous function on $\bar{V}_{0}$ with the uniform norm and define the sequence of linear functionals on this space by the formula

$$
l_{n}\left(V_{0}, \Psi\right)(h)=\frac{1}{n} \sum_{K=0}^{n-1} \int_{V_{0}} \Psi(x) h\left(f^{K}(x)\right) d \nu(x),
$$

where $h \in C\left(\bar{V}_{0}\right)$. It is easy to see that for any $h_{1}, h_{2} \in C\left(\bar{V}_{0}\right)$

$$
\left|l_{n}\left(V_{0}, \Psi\right)\left(h_{1}\right)-l_{n}\left(V_{0}, \Psi\right)\left(h_{2}\right)\right| \leq \max _{x \in V_{0}}\left|h_{1}(x)-h_{2}(x)\right| .
$$

It is easy to see that the family $\left\{l_{n}\right\}$ is relatively compact in the topology of weak * convergence. Let $l\left(V_{0}, \Psi\right)$ be a limit functional. According to Riesz's theorem there exists Borel measure $\mu=\mu\left(l\left(V_{0}, \Psi\right)\right)$, corresponding to $l\left(V_{0}, \Psi\right)$ such that for any continuous function $h \in C\left(\bar{V}_{0}\right)$

$$
l\left(V_{0}, \Psi\right)(h)=\int_{V_{0}} h(x) d \mu(x) .
$$

It is easy to show that the measure $\mu\left(l\left(V_{0}, \Psi\right)\right)$ is normalized, concentrated on $\Lambda$ and $f$-invariant. The main result of this paper is the following theorem.

THEOREM 3. There exists a neighbourhood $V_{0} \subset U_{0}$ of $\Lambda$ with the following property. For any neighbourhood $V_{0}^{\prime} \subset V_{0}$ of $\Lambda$ and any non-negative density $\Psi(x)$ (satisfying (1.5)) any limit measure $\mu=\mu\left(l\left(V_{0}^{\prime}, \Psi\right)\right)$ constructed in the manner described above is a normalized f-invariant Gibbs u-measure.

This assertion means that there exist $f$-invariant Gibbs $u$-measures on $\Lambda$ which can be obtained by iterations of absolutely continuous measures with respect to 
Lebesque measure and concentrated on a neighbourhood of $\Lambda$. In particular the set $\Phi(\Lambda)$ is non-empty. In the case of the set $\Lambda$ being a hyperbolic attractor this assertion was proved earlier by Bowen and Ruelle (cf. $[15,16]$ ). In this case the limit measure $\mu$ is uniquely defined and the map $f \mid \Lambda$ is isomorphic (with respect to $\mu$ ) to a Bernoulli shift.

1.6. We describe another way of constructing Gibbs $u$-measures. Let us fix $x \in \Lambda$ and choose a small open neighbourhood $U_{0}$ of $x$ on the layer $W^{u}(x)$. Let us put

$$
U_{n}=f^{n}\left(U_{0}\right), \quad c_{0}=1, \quad c_{n}=\left[\prod_{K=0}^{n-1} \Phi^{u}\left(f^{K}(x)\right)\right]^{-1}
$$

and define a measure $\tilde{\nu}_{n}$ on $U_{n}$ by the equality

$$
d \tilde{\nu}_{n}(y)=c_{n} \rho_{g^{u}}\left(f^{u}(x), y\right) d \nu^{u}(y), n \geq 0 .
$$

We define a Borel measure $\nu_{n}$ on $\Lambda$ by putting for any Borel set $A \subset \Lambda$

$$
\nu_{n}(A)=\tilde{\nu}_{n}\left(A \cap U_{n}\right), n \geq 0 .
$$

Proposition 3. For any $n \geq 0$ and any Borel set $A \subset \Lambda$ we have

$$
\nu_{n}(A)=\nu_{0}\left(f^{\sim n}(A)\right) .
$$

Let $\mu_{n}=\frac{1}{n} \sum_{K=0}^{n-1} \nu_{K}$. It follows easily from proposition 3 and the Krylov-Bogoljubov theorem that the sequence $\mu_{n}$ is weakly compact and any of its limits is $f$-invariant.

THEOREM 4. Any limiting measure of the sequence $\mu_{n}$ is an f-invariant Gibbs $u$-measure.

Remark. The last assertion admits an important generalization. It is true for attractors which have more general properties than partial hyperbolicity. Namely, it is sufficient to assume, instead of conditions (A2) and (A3), the following conditions:

(A2)' there exists a continuous $f$-invariant contracting (under $f^{-n}$ ) $C^{1}$-foliation $W$ of set $\Lambda$;

$(\mathrm{A} 3)^{\prime} W(x) \subset \Lambda$ for any $x \in \Lambda$;

(A4)' distribution $T W(x)$ satisfies the Hölder condition.

The proof of this fact is the same as the proof of theorem 4 , given in $\$ 2$.

1.7. Sometimes the partial hyperbolicity is formulated in another way. Namely one assumed that the spectrum of the complexification of operator $f_{*}$ is contained in three rings whose radii are $\lambda_{1}^{\prime}, \mu_{1}^{\prime}, \lambda_{2}^{\prime}, \mu_{2}^{\prime}, \lambda_{3}^{\prime}, \mu_{3}^{\prime}$ respectively and (compare with (1.1))

$$
\infty>\lambda_{1}^{\prime} \geq \mu_{1}^{\prime}>\lambda_{2}^{\prime} \geq \mu_{2}^{\prime}>\lambda_{3}^{\prime} \geq \mu_{3}^{\prime}>0, \mu_{1}^{\prime}>1>\lambda_{3}^{\prime} .
$$

We denote the $d f$-invariant distributions on $\Lambda$ corresponding to these rings by $E^{u}$, $E^{0}$ and $E^{s}$ respectively. 
THEOREM 5. Let us assume that the distribution $E^{0}$ is integrable (cf. [14]) and the layers of the corresponding foliation $W^{0}$ are compact subsets of $\Lambda$. Then for any $\varepsilon>0$ there exists a neighbourhood $\mathbb{U} \subset D$ iff $\left.{ }^{(1+\alpha}\right)(U, M)$ of $f$ such that

(1) any diffeomorphism $g \in \mathbb{U}$ has a partially hyperbolic attractor $\Lambda_{g} \subset U$;

(2) the spectrum of the complexification of the operator $g_{*}$ in $\Gamma^{0}\left(T \Lambda_{g}\right)$ is contained in three rings whose radii are equal to $\lambda_{i, g}, \mu_{i, g}, i=1,2,3$ respectively, where

$$
\infty>\lambda_{1,8} \geq \mu_{1,8}>\lambda_{2,8} \geq \mu_{2,8}>\lambda_{3,8} \geq \mu_{3,8}>0, \quad \mu_{1,8}>1>\lambda_{1,8} ;
$$

(3) the distribution $E_{\mathrm{g}}^{0}$, corresponding to the middle component of the spectrum, is integrable and the layers of the corresponding foliation $W_{8}^{0}$ are compact subsets in $\Lambda_{8}$;

(4) there exists a homeomorphism $h_{g}: \Lambda \rightarrow \Lambda_{g}$ such that for every $x \in \Lambda_{g}$

$$
\rho\left(h_{\mathrm{B}}(x), x\right) \leq \varepsilon ;
$$

(5) $h_{g}\left(W^{0}(x)\right)=W_{g}^{0}\left(h_{g}(x)\right)$ for any $x \in \Lambda$.

\section{Proofs}

2.1. Proof of proposition 1. (1) Let $V_{0} \subset U_{0}$ be a neighbourhood of $\Lambda$, then by the condition (A3).

$$
\Lambda \subset \bigcap_{n \geq 0} f^{n}\left(V_{0}\right) \subset \bigcap_{n \geq 0} f^{n}\left(U_{0}\right)=\Lambda .
$$

(2) Let $\Lambda$ be a compact $f$-invariant set and $\Lambda^{\prime} \subset U_{0}$, then

$$
\Lambda^{\prime} \subset \bigcap_{n \geq 0} f^{n}\left(U_{0}\right)=\Lambda .
$$

(3) Assume that the converse is true. Then for any $\varepsilon>0$ there exist $x \in \Lambda$ and $y \in W_{\varepsilon}^{u}(x)$ such that $y \notin \Lambda$. The condition (A3) implies that $f^{n}(y) \in U_{0}$ for $n \geq 0$. If $\varepsilon>0$ is small enough then it follows from the definition of sets $W_{\varepsilon}^{u}(x)$, compactness of $\Lambda$ and the condition (A2) that $f^{n}(y) \in U_{0}$ for $n \leq 0$. But this contradicts the local maximality of $\Lambda$. The fact that the sets $W^{u}(x)$ form a continuous $f$-invariant $C^{1}$-foliation contracting under $f^{-n}$ follows from condition (A2.)

2.2. Proof of proposition 2. As the function $\phi$ is continuous and does not vanish on $\Lambda$ we have

$$
\min _{x \in \Lambda} \phi(x)>0 \quad \text { if } \phi>0 .
$$

As the function $\phi$ satisfies Hölder's condition we get using assertion 3 of proposition 1 that for any $K \geq 0, x \in \Lambda, y \in \overline{B^{u}(x, r)}$

$$
\left|\phi\left(f^{-K}(x)\right) / \phi\left(f^{-K}(y)\right)-1\right| \leq C\left(\mu_{1}\right)^{\alpha K} r^{\alpha} .
$$

Assertions 1 and 2 follow from this inequality. Assertion 3 is obvious

2.3. Proof of theorem 1 is essentially a simple modification of the proof given in [4] (cf. also $[7,13,6])$ and therefore is omitted.

2.4. Proof of theorem 2 is a modification of the proof given in [10]. Let $\Pi=\Pi(x, W, r)$ be a $\Lambda$-rectangle at $x \in \Lambda$. We define an open set $U(y)$ for every $y \in W \cap \Lambda$ in the following way: $z \in U(y)$ if there exists a finite sequence of sets

$$
A_{i}=f^{-n_{i}}\left(B^{u}\left(y_{i}, r\right)\right) \quad\left(y_{i} \in W \cap \Lambda, n_{i} \geq 0, i=1, \ldots, p\right)
$$


such that

(1) $A_{1}=B^{u}(y, r)$;

(2) $A_{i} \cap A_{i-1} \neq \varnothing \quad(1<i \leq p)$;

(3) $z \in A_{p}$.

One can prove, repeating the arguments given in $[10]$ that the set

$$
\tilde{\Pi}=\bigcup_{y \in W \cap \Lambda} U(y)
$$

has the following property; if $f^{-n}(U(y)) \cap \tilde{\Pi} \neq \varnothing$ for some $y \in W \cap \Lambda$ and an integer $n \geq 0$ then

$$
f^{-n}(U(y)) \subset U(z) \text { for some } z \in W \cap \Lambda \text {. }
$$

It is clear that the set $U(y), y \in W$, is open in $W^{u}(y)$ and connected. We shall show that $\nu^{u}(\partial U(y))=0$. Let $\hat{\Pi}=\Pi(x, W, R)$ be a $\Lambda$-rectangle at $x$ and $R>r$. Let also $\hat{U}(y)$ and $U(y)$ be the sets constructed as pointed out above. Obviously we have

$$
\hat{U}(y) \supset U(y) \text { and } \nu^{u}(\hat{U}(y) \backslash U(y)) \leq c(R-r),
$$

where $c>0$ is a constant independent on $R$ and $r$,

As the set $\tilde{\Pi}$ is open in $\Lambda$ the set

is also open in $\Lambda$. Let

$$
\Lambda^{\prime}=\bigcup_{-\infty<n<\infty} f^{n}(\tilde{\Pi})
$$

$$
\Lambda^{\prime \prime}=\left\{w \in \Lambda^{\prime}: f^{n}(w) \in \tilde{\Pi} \quad \text { for some } n>0\right\} .
$$

For $w \in \Lambda^{\prime \prime}$ we denote by $N(w), w \in \Lambda^{\prime}$ the set of all integers $n>0$ such that $f^{n}(w) \in \tilde{\Pi}$ and put

$$
\tilde{U}(w)=\bigcup_{n \in N(w)} f^{-n}\left(U\left(f^{n}(w)\right)\right) .
$$

Since for every $\mu \in \Phi(\Lambda), \mu\left(\Lambda^{\prime} \backslash \Lambda^{\prime \prime}\right)=0$ the construction may be extended to the rest of the set $\Lambda^{\prime}$ in a more or less arbitrary manner.

The following properties of the set $\tilde{U}(w)$ follow from what was stated above.

(1) $\tilde{U}(w)=U(w)$ where $w \in \tilde{\Pi}$;

(2) $f^{-n}\left(\tilde{U}\left(w_{1}\right)\right) \subset \tilde{U}\left(w_{2}\right)$, if $w_{2}=f^{-n}\left(w_{1}\right)\left(w_{1}, w_{2} \in \Lambda^{\prime}, n \geq 0\right)$;

(3) the sets $\tilde{U}(w)$ are open and connected;

(4) diam $\tilde{U}(w) \leq$ const independently on $w$;

(5) $\nu^{u}(\partial \tilde{U}(w))=0$.

Properties (1) and (2) imply that the sets $\tilde{U}(w)$ form a partition of $\Lambda^{\prime}$ which we shall denote by $\xi$. As $\xi$ is continuous it is measurable with respect to any Borel measure $\mu$ on $\Lambda$. Assertions 2 and 3 follow from properties (1)-(5) of the sets $\tilde{U}(w)$, and assertion 4 is proved with the help of assertion 4 of proposition 1 as in [6] (cf. also [8]). If $\mu$ is a Borel measure then there exists a $\Lambda$-rectangle $\Pi$ which is open in $\Lambda$ such that $\mu(\Pi)>0$. Therefore $\mu\left(\Lambda^{\prime}\right)>0$ and thus $\mu\left(\Lambda^{\prime}\right)=1$ because $\Lambda^{\prime}$ is $f$-invariant and $\mu$ is ergodic. The last part of assertion 5 can be stated as in [6] (cf. also [8]). 
2.5. Proof of theorem 3 consists of two parts. The first one (cf. lemmas 1-10) is auxiliary but also has an independent interest. It is unknown whether one can continue the foliation $W^{u}$ of $\Lambda$ up to a ' $f$-invariant expansive continuous and absolutely continuous' foliation in the whole neighbourhood $U_{0}$ of $\Lambda$ (cf. $\S 3$ for details). However, one can prove a weaker version, namely one can continue the family of local manifolds

$$
\left\{\exp _{x}^{-1} W_{\mathrm{loc}}^{u}(x), x \in \Lambda\right\}
$$

up to a continuous family

$$
\mathbb{W}=\left\{W(x), x \in U_{0}\right\},
$$

where each $W(x)$ is a smooth submanifold in $T_{\mathrm{x}} M$ (we have $W(x)=\exp _{x}^{-1} W_{\mathrm{loc}}^{u}(x)$ for any $x \in \Lambda$ ). The family $W$ is invariant (cf. lemma 7) and contracting with respect to $f^{-1}$ (cf. lemma 8). Moreover, the submanifolds

$$
\tilde{W}\left(x_{i}\right)=\exp _{x_{i}} W\left(x_{i}\right)
$$

where $x_{i}, i=1,2$, are two points in a local transversal manifold $W$ do not intersect each other (cf. lemma 9). This last fact allows 'the expansion' of every $\Lambda$-rectangle to a 'full' rectangle $\tilde{\Pi}$, defined by the formula

$$
\tilde{\Pi}=\bigcup_{y \in W} \tilde{W}(y) .
$$

The partition $\tilde{\xi}$ of $\tilde{\Pi}$ into sets $\tilde{W}(x)$ is absolutely continuous (cf. lemma 10 ).

After these preparatory steps we pass to the second part of the proof (cf. lemmas 11-13). The main idea is to show that the sequence of conditional measures for the measures $\mu_{n}$ (corresponding to the functionals $l_{n}$ ) with respect to the partition $\tilde{\xi}$ converges as $n \rightarrow \infty$.

Let $U_{0}$ be a neighbourhood of $\Lambda$ appearing in condition A3 and $U_{n}=f^{n}\left(U_{0}\right)$. From $U_{1} \subset U_{0}$ we have $U_{n} \subset U_{n-1}$ for any $n>0$. Therefore, for any $n>0$

$$
\alpha_{n}=\rho\left(\partial U_{n}, \partial U_{n-1}\right)>0 .
$$

Let $x \in \Lambda$ and $q>0$. Suppose that

$$
\begin{gathered}
B^{s 0}(x, q)=\exp _{x}\left\{y \in E^{s 0}(x):\|y\|<q\right\}, \\
U(q)=\bigcup_{x \in \Lambda} B^{s 0}(x, q) .
\end{gathered}
$$

The following statement follows directly from proposition 1 .

LEMMA 1. (1) There exists $q_{0}>0$ such that for any $q \in\left(0, q_{0}\right]$ the set $U\left(q_{0}\right)$ is an open neighbourhood of $\Lambda$ which is contained in $U_{0}$

(2) For every $q \in\left[0, q_{0}\right]$ there exists an integer $m=m(q)>0$ such that $\bar{U}_{n} \subset U(q)$ for $n \geq m(q)$.

We denote by $d$ the distance in $T_{\mathrm{x}} M$, induced by the Riemannian metric. Let us fix two constants $r>0, C>0$ and $y \in U_{0}$. We call an open smooth submanifold 
$V(y)$ in $T_{y} M$ a local $(r, C)$-manifold at $y$ if

(L1) $0 \in V(y)$;

(L2) $\|z\| \geq r$ for every $z \in \partial V(y)$;

(L3) for any $y_{1}, y_{2} \in \tilde{V}(y)=\exp _{y} V(y)$

$$
d\left(T_{y_{1}} \tilde{V}(y), T_{y_{2}} \tilde{V}(y)\right) \leq C
$$

(L4) if $x \in \Lambda, y \in B^{s 0}(x, q)$ then

$$
d\left(T_{y} \tilde{V}(y), E^{u}(x)\right) \leq C .
$$

LEMMA 2. There exist $q_{1} \in\left(0, q_{0}\right], r_{0}>0, C_{0}>0$ such that for any $q \in\left(0, q_{1}\right], r \in\left(0, r_{0}\right]$, $C \in\left(0, C_{0}\right]$ and any local $(r, C)$-manifold $V(y)$ at $y \in \bar{U}_{m}(m=m(q)$, cf. lemma 1) the inclusion

takes place.

$$
\tilde{V}(y) \subset U_{0}
$$

The proof follows directly from proposition 1 and lemma 1.

Lemma 3. There exist $q_{2} \in\left(0, q_{1}\right], r_{1} \in\left(0, r_{0}\right], C \in\left(0, C_{0}\right]$ such that for any $q \in\left(0, q_{2}\right]$, $r \in\left(0, r_{1}\right]$ and any local $(r, C)$-manifold at $y \in \bar{U}_{m}, m=m(q)$ (cf. lemma 1) the following inclusion

$$
\exp _{f(y)}^{-1} \circ f \circ \exp _{y} V(y) \supset \hat{V}(f(y))
$$

holds where $\hat{V}(f(y))$ is a local $(r, C)$-manifold at $f(y)$.

Proof. Let us choose $x \in \Lambda$ in such a way that $y \in B^{s 0}(x, q)$. We have

$$
\exp _{f(x)}^{-1} \circ f \circ \exp _{x}(u, v)=\left(A_{x} u+g_{1 x}(u, v), B_{x} v+g_{2 x}(u, v)\right),
$$

where $u, v$ lie in small neighbourhoods $U, V$ of the origin in the spaces $E^{u}(x)$ and $E^{s 0}(x)$ respectively,

$$
A: E^{u}(x) \rightarrow E^{u}(f(x)), \quad B: E^{s 0}(x) \rightarrow E^{s 0}(f(x))
$$

are linear operators,

$$
g_{x}=\left(g_{1 x}, g_{2 x}\right): U \times V \rightarrow T_{f(x)} M
$$

are smooth maps and

$$
g_{x}(0,0)=(0,0), \quad d g_{x}(0,0)=(0,0) .
$$

It follows from (1.2) that

$$
\left\|A_{x}\right\| \geq \mu_{1}, \quad\left\|B_{x}\right\| \leq \lambda_{2} .
$$

It is not difficult to show, using (2.1), (2.2), (2.3), the properties L1-L4 and the arguments of the 'hyperbolic' character, that the submanifold

$$
W(y)=\exp _{f(y)}^{-1} \circ f \circ \exp _{y} V(y) \subset T_{f(y)} M
$$

has the following properties:

(1) $0 \in W(y)$;

(2) $\|z\| \geq(1-\varepsilon)(1-C)\left(\mu_{1}-\varepsilon-\varepsilon C\right) r \quad$ where $\varepsilon=\varepsilon(q, r) \rightarrow 0 \quad$ as $\quad q, r \rightarrow 0$ and $z \in \partial W(y)$

(3) for any $y_{1}, y_{2} \in \tilde{W}(y)=\exp _{y} W(y)$

$$
d\left(T_{y_{1}} \tilde{W}(y), T_{y_{2}} \tilde{W}(y)\right) \leq(1+\varepsilon)\left(\left(\lambda_{2}+\varepsilon\right) /\left(\mu_{1}-\varepsilon\right)\right) C+\delta(\varepsilon) ;
$$


where $\delta(\varepsilon) \rightarrow 0$ as $\varepsilon \rightarrow 0$ (and consequently as $q, r \rightarrow 0)$;

(4) $d\left(T_{y} \tilde{W}(y), E^{u}(f(x))\right) \leq(1+\varepsilon)\left(\left(\lambda_{2}+\varepsilon\right) /\left(\mu_{1}-\varepsilon\right)\right) C+\delta(\varepsilon)$.

Property (2) implies that for any small enough $q, r, C$ and any $z \in \partial W(y)$

$$
\|z\| \geq r \text {. }
$$

As it follows from property (3) for given $C>0$ and any small enough $q, r$

$$
d\left(T_{y_{1}} \tilde{W}(y), T_{y_{2}} \tilde{W}(y)\right) \leq C,
$$

where $y_{1}, y_{2} \in \tilde{W}(y)$. Now let $z$ be a point such that $f(y) \in B^{s 0}(z, q)$. It is easy to see that

$$
\rho(f(y), z) \leq K_{1} q,
$$

where $K_{1}>0$ is a constant which is independent on $q, r, C$. As $f(y) \in \bar{U}_{m+1} \subset U_{m}$ we have

$$
\rho(f(y), f(x)) \leq K_{2} q,
$$

where $K_{2}>0$ is a constant, which is independent on $q, r, C$. It follows from (2.6), (2.7) and the triangle inequality that

$$
\rho(f(x), z) \leq\left(K_{1}+K_{2}\right) q .
$$

As the distribution $E^{u}$ satisfies Hölder's condition on $\Lambda$ (cf. [3]) and $f(x), z \in \Lambda$ we have, using (2.8)

$$
d\left(E^{u}(z), E^{u}(f(x))\right) \leq K_{3} q^{\alpha},
$$

where $K_{3}>0$ and $\alpha \in(0,1]$ are constants. It follows from (2.9) and property (4) that for every small enough $q, r$

$$
d\left(T_{y} \tilde{W}(y), E^{u}(z)\right) \leq C .
$$

Now the assertion which we need follows from (2.4), (2.5), (2.10).

Let us fix $q \in\left(0, q_{2}\right], r \in\left(0, r_{1}\right]$, choose $m=m(q)$ in accordance with lemma 1 and consider the set $U_{m}$. Let

$$
P_{m}=\left\{y \in \bar{U}_{m}: \rho\left(y, \partial U_{m}\right) \leq 100^{-1} \alpha_{m+1}\right\} .
$$

LEMMA 4. There exist $q_{3} \in\left(0, q_{2}\right], r_{2} \in\left(0, r_{1}\right]$ such that for any $q \in\left(0, q_{3}\right], r \in\left(0, r_{2}\right]$ the following is valid:

(1) there exists a smooth distribution $E(y)$ in $P_{m}(m=m(q))$ such that

$$
d\left(E(y), E^{u}(x)\right) \leq C,
$$

where $C>0$ is the constant, which appeared in lemma 3 , and $x \in \Lambda$ is any point for which $y \in B^{s 0}(x, q)$;

(2) the submanifold

$$
W(y)=\{z \in E(y):\|z\| \leq r\}
$$

is a local $(r, C)$-manifold at $y$.

Proof. As the distribution $E^{u}$ is continuous we can extend it up to a continuous distribution $\tilde{E}(y)$, in a neighbourhood $Q$ of $\Lambda$. Let us take $q_{3} \in\left(0, q_{2}\right]$ so small that $U_{m} \subset Q\left(m=m\left(q_{3}\right)\right)$. Now we can choose as $E(y)$ any smooth distribution close enough to $\tilde{E}(y)$ in $P_{m}$. 
The second assertion follows directly from the first one. Let us fix $q \in\left(0, q_{3}\right]$, $r \in\left(0, r_{2}\right]$ and let $C$ be the constant which appeared in lemma 3 . It is easy to see that the following assertion is valid.

LEMMA 5. There exists a family of local $(r, C)$-manifolds

$$
\vee=\left\{V(y), y \in \bar{U}_{m} \mid U_{m+1}\right\}
$$

satisfying the following conditions:

(1) $V(y)=W(y)$ for any $y \in P_{m}$ (cf. lemma 4);

(2) $V(y)=\hat{W}(y)$ for any $y \in f\left(P_{m}\right)$ where as in lemma 3

$$
\hat{W}(y)=\exp _{f(y)}^{-1} \circ f \circ \exp _{y} W(y) ;
$$

(3) The submanifold $V(y)$ depends smoothly on $y \in \bar{U}_{m} \mid U_{m+1}$

(4) $\left(\bigcup_{y \in \overline{U_{m}} \backslash U_{m+1}} \exp _{y} V(y)\right) \cap \Lambda=\varnothing$.

We consider the sequence of the families of local manifolds

$$
\mathbb{V}^{n}=\left\{V^{n}(y), y \in U_{m}\right\}
$$

defined in the following way:

(1) $\mathbb{V}^{0}=\mathbb{V}$ (cf. lemma 5);

(2) $V^{n}(y)=V^{n-1}(y)$ for any $y \in \bar{U}_{m} \mid U_{m+n}$;

(3) $V^{n}(y)=\hat{V}(y)$, where the local manifold $\hat{V}(y)$ is constructed with the help of the local manifold $V^{n-1}\left(f^{-1}(y)\right)$ in accordance with lemma 3 .

We shall say that a family of local manifolds $\mathbb{V}=\left\{V(y), y \in U_{0}\right\}$ satisfies Hölder's condition with parameters $(R, K, \alpha), R>0, K>0, \alpha \in(0,1]$ if for any $x \in \Lambda, y, y_{1}$, $y_{2} \in B^{s 0}(x, q)$ the following conditions hold:

(1) The submanifold $\exp _{x}^{-1} \exp _{y} V(y)$ contains the graph of a smooth function

$$
\phi(x, y): B^{u}(R) \rightarrow E^{s 0}(x) \text { where } B^{u}(R)
$$

is the ball in $E^{u}(x)$ with its centre at the origin and radius $R$;

$$
\max _{z \in B^{u}(R)}\left\|\frac{d \phi\left(x, y_{1}\right)(z)}{d z}-\frac{d \phi\left(x, y_{2}\right)(z)}{d z}\right\| \leq K \rho\left(y_{1}, y_{2}\right)^{\alpha} .
$$

LEMMA 6. There exists $q_{4} \in\left(0, q_{3}\right]$ such that for any $q \in\left(0, q_{4}\right], r \in\left(0, r_{3}\right), \varepsilon \in\left(0, \varepsilon_{0}\right]$ the families $\mathbb{V}^{n}, n=0,1,2, \ldots$ have the following properties:

(1) The submanifold $V^{n}(y)$ depends smoothly on $y \in \bar{U}_{m}$;

(2) The family $\mathbb{V}^{n}$ satisfies Hölder's condition with parameters $(R, K, \alpha)$, independent on $n$;

Proof. (1) follows from lemma 5 and the definition of the family $\mathbb{V}^{n}$.

(2) Assertion 3 of lemma 5 implies that the family $\mathbb{V}^{0}=\mathbb{V}$ satisfies Hölder's condition with parameters $(R, K, \alpha)$ for some $R>0, K>0$. We assume that the family $\mathbb{V}^{n-1}$ satisfies Hölder condition with some parameters $(R, K, \alpha)$. Let $x \in \Lambda$, $y \in B^{s 0}(x, q) \cap \bar{U}_{m+n}$. We take $\hat{x} \in \Lambda$ so that $f^{-1}(y) \in B^{s 0}(\hat{x}, q)$. Then we get

$$
\rho\left(\hat{x}, f^{-1}(y)\right) \leq K_{4} q
$$


where $K_{4}>0$ is a constant, independent on $\hat{x}, y, n$. It is easy to see that

$$
\rho\left(f^{-1}(x), f^{-1}(y)\right) \leq K_{5} q,
$$

where $K_{s}>0$ is a constant independent on $x, y, n$. It follows from (2.11), (2.12) and the triangle inequality that

$$
\rho\left(\hat{x}, f^{-1}(x)\right) \leq K_{6} q,
$$

where $K_{6}>0$ is a constant. We get from this and the inductive assumption that the submanifold

$$
\exp _{f^{-1}(x)}^{-1}\left(\exp _{f^{-1}(y)} V^{n-1}\left(f^{-1}(y)\right)\right)
$$

contains the graph of a function

$$
\hat{\phi}\left(f^{-1}(x), f^{-1}(y)\right): B^{u}(\hat{R}) \rightarrow E^{s 0}\left(f^{-1}(x)\right),
$$

where

$$
\hat{R} \geq R(1-\delta(q))
$$

and $\delta(q) \rightarrow 0$ when $q \rightarrow 0$. It follows from (2.1), (2.2) and (2.3) that the submanifold

$$
\exp _{x}^{-1}\left(f\left(\exp _{f^{-1}(y)} V^{n-1}\left(f^{-1}(y)\right)\right)\right)
$$

contains the graph of the function

where

$$
\tilde{\phi}(x, y): B^{u}(\tilde{R}) \rightarrow E^{s 0}(x),
$$

$$
\tilde{R} \geq\left(\mu_{1}-\varepsilon-C \varepsilon\right) \hat{R} .
$$

(Number $C$ is taken in accordance with lemma 3.) Inequalities (2.13) and (2.14) imply that for any small enough $q$

$$
\tilde{R} \geq R \text {. }
$$

Now let $y_{1}, y_{2} \in B^{s 0}(x, q) \cap \bar{U}_{m+n}$. One can show repeating the above arguments that

$$
\begin{aligned}
& \max _{z \in B^{u}(\tilde{R})}\left\|\frac{d \tilde{\phi}\left(x, y_{1}\right)(z)}{d z}-\frac{d \tilde{\phi}\left(x, y_{2}\right)(z)}{d z}\right\| \\
& \leqq \leqq(1+\varepsilon)\left(\frac{\left(\lambda_{2}+\varepsilon\right)^{1+\alpha}}{\mu_{1}-\varepsilon}\right) K \rho\left(y_{1}, y_{2}\right)^{\alpha}+K_{6}^{\prime} \\
& \leq K \rho\left(y_{1}, y_{2}\right)^{\alpha},
\end{aligned}
$$

where $K_{6}^{\prime}>0$ is a constant and $\varepsilon=\varepsilon(q)$. The last inequality is valid if we take numbers $q>0, \alpha>0$ so small that in view of (1.2)

$$
\gamma=\left(\lambda_{2}+\varepsilon\right)^{1+\alpha} /\left(\mu_{1}-\varepsilon\right)<1
$$

and number $K$ so large that

$$
K \geq K_{6}^{\prime} /(1-\gamma) \text {. }
$$

The following assertion is the consequence of lemma 6 , the definition of the families $\mathbb{V}^{n}$ and the properties of foliation (cf. [9]).

LemMA 7. There exist $q>0, r>0, C>0, R>0, K>0$, and a family of local $(r, C)$-manifolds

$$
\mathbb{W}=\left\{W(y), y \in U_{m}\right\}(m=m(q))
$$


such that:

(1) the submanifold $W(y)$ depends smoothly on $y \in U_{m} \backslash \Lambda$;

(2) the family $W$ satisfies Hölder's condition with parameters $(R, K, \alpha)$;

(3) $\exp _{x} W(x) \subset W^{u}(x)$ for each $x \in \Lambda$;

(4) $f\left(\exp _{x} W(x)\right) \supset \exp _{f(x)} W(f(x))$ for each $x \in \Lambda$;

We also need three important properties of the family $\mathbb{W}$, stated in lemmas 8 , 9,10 .

LEMMA 8. There exists $K_{7}>0$ such that for any $y \in \bar{U}_{m}, z \in \exp _{y} W(y)$ and any $l \geq 0$ for which $f^{-s}(y) \in \bar{U}_{m}, s=0,1, \ldots, l$ the following inequality holds

$$
\rho\left(f^{-l}(y), f^{-l}(z)\right) \leq K_{7}\left(\mu_{1}-\varepsilon\right)^{-l} \rho(y, z) .
$$

Proof. If $y \in \Lambda$ then the result which we need follows from assertion 3 of lemma 7 and assertion 3 of proposition 1. If $y \in \bar{U}_{m} \backslash \Lambda$ then inequality (2.15) is the consequence of assertion 4 of lemma 7 , the definition of the local manifold (we remark that parameters $r, C$, appearing in this definition, are independent on $y$ ) and assertion 3 of proposition 1.

LEMMA 9. Under the conditions of lemma 7 there exists $r_{0} \in(0, r]$ such that for any $x \in \Lambda, y_{1}, y_{2} \in B^{s 0}(x, q)$

$$
B_{W}\left(y_{1}, r_{0}\right) \cap B_{W}\left(y_{2}, r_{0}\right)=\varnothing,
$$

where $B_{W}\left(y, r_{0}\right)$ is the ball on the submanifold $\tilde{W}(y)=\exp _{y} W(y)$ with a centre at $y$ and radius $r_{0}$.

Proof. Let us fix small $\beta>0, \delta>0$ and an integer $n>0$ satisfying certain restrictions which will be specified later. We choose $m=m(\delta)$ such that $\bar{U}_{m}$ lies in the $\delta$-neighbourhood of $\Lambda$ and consider the set $U_{m+n}$. Let us put $F=f^{-n}$. Let $x \in \Lambda$, $y \in B^{s 0}(x, q) \cap U_{m+n}$ and $y \notin \Lambda$. We denote by $K(y)$ the smallest integer for which

$$
F^{K(y)}(y) \in U_{m+n} \mid U_{m+2 n} .
$$

Such a number obviously exists, is uniquely defined and is strictly positive. Let $E(y)$ be the subspace of $T_{y} M$, having the same dimension as $E^{s 0}(x)$ and

$$
d\left(E(y), E^{s 0}(x)\right) \leq \beta .
$$

We consider the point $w=F(y)$ and assume that $K(y)>0$. Then

$$
w \in B^{s 0}(z, q) \cap U_{m+n} \quad \text { for some } z \in \Lambda \text {. }
$$

One can show using (2.1), (2.2), (2.3), (2.16) and the fact that

that

$$
E^{s 0}(F(x))=d F\left(E^{s 0}(x)\right)
$$

$$
d\left(d F(E(y)), E^{s 0}(F(x))\right) \leq K_{8}\left(\frac{\lambda_{2}+\varepsilon}{\mu_{1}-\varepsilon}\right)^{n} \beta,
$$

where $K_{8}>0$ is a constant. As the distribution $E^{u}(x)$ satisfies Hölder's condition the distribution $E^{s 0}(x)$ also satisfies this condition (cf. [3]). Therefore the triangle inequality implies

$$
d\left(E^{s 0}(F(x)), E^{s 0}(z)\right) \leq K_{9} \rho(F(x), z)^{\alpha} \leq K_{9}[\rho(F(x), w)+\rho(w, z)]^{\alpha},
$$


where $K_{9}>0$ is a constant and $\alpha \in(0,1]$. Our assumptions concerning the points $y, z$ and $w$ imply that

$$
\begin{gathered}
\rho(w, z) \leq K_{10} \delta, \\
\rho(F(x), w)=\rho(F(x), F(y)) \leq K_{10}\left(K_{11}\right)^{n} \delta,
\end{gathered}
$$

where $K_{10}>0$ and $K_{11}>0$ are constants and $K_{11}<1$. It follows from (2.17)-(2.20) that

$$
d\left(d F(E(y)), E^{s 0}(z)\right) \leq K_{8}\left(\frac{\lambda_{2}+\varepsilon}{\mu_{1}-\varepsilon}\right)^{n} \beta+K_{9}\left[K_{10}\left(\left(K_{11}\right)^{n}+1\right) \delta\right]^{\alpha} .
$$

The last expression is less than $\beta$ provided $n$ is large enough and $\delta=\delta(n, \beta)$ is small enough. Repeating the previous arguments and making the induction in $K=K(y)$ one can prove the following: if $l \in[0, K(y)]$ and $z \in \Lambda$ are such that

$$
F^{l}(y) \in B^{s 0}(z, q) \cap U_{m+n}
$$

then

$$
d\left(d F^{l}(E(y)), E^{s 0}(z)\right) \leq \beta .
$$

Now let $x \in \Lambda, y \in B^{s 0}(x, q) \cap U_{m+n}$ and $y \notin \Lambda$. If $\delta$ is small enough then the subspace $E(y)=T_{y} B^{s 0}(x, q)$ satisfies (2.16). Therefore we get from the above mentioned arguments that for any $\varepsilon>0$ there exists $\rho>0$ with the following property: the open ball $B(w)$ on the submanifold

$$
f^{-(K(y)-1) n}\left(U_{m+2 n} \cap B^{s 0}(x, q)\right)
$$

with its centre at $w=f^{-(K(y)-1) n}(y)$ and radius $\rho$ is $\varepsilon$-near in $C^{1}$-topology to an open set $U \subset B^{s 0}(z, q)$, where $z \in \Lambda$ is a point such that $w \in B^{s 0}(z, q) \cap U_{m+2 n}$. Without loss of generality one can assume that

$$
0<\rho \leq \min \left\{\alpha_{m+n}, \alpha_{m+2 n}\right\} .
$$

It follows from what was told above and assertion 1 of lemma 7 that there exists $r_{0}^{(1)}>0$ such that for any $x \in \Lambda, y \in B^{s 0}(x, q) \cap B(w)$

$$
B_{W}\left(y, r_{0}^{(1)}\right) \cap B_{W}\left(w, r_{0}^{(1)}\right)=\varnothing .
$$

Moreover, it follows from assertion 1 of lemma 7 that there exists $r_{0}^{(2)}>0$ such that for any $x \in \Lambda, y_{1}, y_{2} \in B^{s 0}(x, q) \backslash U_{m+2 n+1}$

$$
B_{W}\left(y_{1}, r_{0}^{(2)}\right) \cap B_{W}\left(y_{2}, r_{0}^{(2)}\right)=\varnothing .
$$

Therefore it is sufficient to prove the existence of $r_{0}^{(3)}>0$ such that for any $x \in \Lambda$, $y_{1}, y_{2} \in B^{s 0}(x, q) \cap U_{m+n}$

$$
B_{W}\left(y_{1}, r_{0}^{(3)}\right) \cap B_{W}\left(y_{2}, r_{0}^{(3)}\right)=\varnothing .
$$

If $y_{1}, y_{2} \in \Lambda$ then (2.22) follows from proposition 1. Therefore we assume that for any $r_{0}^{(3)}$ and some $x \in \Lambda, y_{1}, y_{2} \in B^{s 0}(x, q) \cap U_{m+n}, y_{1} \notin \Lambda$ the intersection

$$
B_{W}\left(y_{1}, r_{0}^{(3)}\right) \cap B_{W}\left(y_{2}, r_{0}^{(3)}\right)
$$

contains the point $z$. If $n$ is large enough and $r_{0}^{(3)}$ is small enough then from lemma 8

$$
\rho\left(f^{-K\left(y_{1}\right) n}\left(y_{1}\right), \quad f^{-K\left(y_{2}\right) n}(z)\right) \leq r_{0}^{(1)}
$$


and

$$
\rho\left(f^{-K\left(y_{1}\right) n}\left(y_{2}\right), \quad f^{-K\left(y_{1}\right) n}(z)\right) \leq r_{0}^{(1)}
$$

Moreover

$$
\begin{aligned}
\rho\left(f^{-K\left(y_{1}\right) n}\left(y_{1}\right), f^{-K\left(y_{1}\right) n}\left(y_{2}\right)\right) \leq & \rho\left(f^{-K\left(y_{1}\right) n}\left(y_{1}\right), f^{-K\left(y_{1}\right) n}(z)\right) \\
& +\rho\left(f^{-K\left(y_{1}\right) n}(z), f^{-K\left(y_{1}\right) n}\left(y_{2}\right)\right) \leq \rho .
\end{aligned}
$$

As $f^{-K\left(y_{1}\right) n}\left(y_{1}\right) \in U_{m+n} \mid U_{m+2 n}$ the last inequality is impossible in view of (2.21).

Let us put for $x \in \Lambda$

$$
\Pi(x)=\bigcup_{y \in B^{s \mathbf{0}}(x, q) \cap \bar{U}_{m}} \tilde{W}(y),
$$

where $\tilde{W}(y)=B_{W}\left(y, r_{0}\right)$ and we denote by $\xi(x)=\xi(\Pi, x)$ the partition of $\Pi(x)$ into the sets $\tilde{W}(y)$. Let $n \geq 0$ and

$$
\Pi_{n}(x)=\bigcup_{y \in B^{s 0}(x, q) \cap U_{m+n}} \tilde{W}(y), \quad P_{n}(x)=f^{-n}\left(\Pi_{n}(x)\right) .
$$

We denote by $\xi_{n}(x)$ the partition of $\Pi_{n}(x)$ into the sets $\tilde{W}(y), y \in B^{s 0}(x, q) \cap U_{m+n}$ and $\eta_{n}(x)=f^{-n} \xi_{n}(x)$. It is easy to see that $\eta_{n}(x)$ is measurable with respect to any Borel measure $\mu$ on $P_{n}(x)$. One can prove also the following assertion, repeating the arguments, given in [2, (cf. p. 151] and using lemmas 7, 8, 9 .

LEMMA 10. The partition $\eta_{n}(x)$ is absolutely continuous (with respect to Riemannian volume); more exactly, for any Borel set $A \subset P_{n}(x)$ for which $\nu(A)>0$ the equality

$$
\nu(A)=\int_{P_{n}(x) / \eta_{n}(x)} d \nu_{n, x}(y) \int_{C_{\eta_{n}(x)}(y)} \rho_{y}^{n}(z) \chi_{A}(z) d \nu_{n, x, y}(z)
$$

is valid. Here $\nu_{n, x}$ is the measure in factor-space $P_{n}(x) / \eta_{n}(x), \nu_{n, x, y}$ is the measure on $f^{-n}(\tilde{W}(y))$, induced by the Riemannian metric, $\chi_{A}(z)$ is the characteristic function of $A, \rho_{y}^{n}(z)$ is 'the density function'. It is a continuous function, defined on the set

$$
C_{\eta_{n}(x)}(y)=f^{-n}(\tilde{W}(y)), y \in B^{s 0}(x, q) \cap U_{m+n}
$$

and satisfying the condition

$$
\left|\rho_{y}^{n}(z)-1\right| \leq K_{12}\left(\frac{\lambda_{2}+\varepsilon}{\mu_{1}-\varepsilon}\right)^{n}
$$

for any $y \in B^{s 0}(x, q) \cap U_{m+n}, z \in C_{\eta_{n}(x)}(y) .\left(K_{12}>0\right.$ is a constant $)$.

We fix $x \in \Lambda$ and denote

$$
\begin{gathered}
\Pi_{n}=\Pi_{n}(x), \quad \xi=\xi(x), \quad \xi_{n}=\xi_{n}(x), \\
\eta_{n}=\eta_{n}(x), \quad X=B^{s 0}(x, q) \cap U_{m}, \quad X_{n}=B^{s 0}(x, q) \cap U_{m+n} .
\end{gathered}
$$

Also let $n(y), y \in X$ be the integer such that $f^{-K}(y) \in U_{m}$ for $K=0,1, \ldots, n(y)$ and $f^{-(n(y)+1)}(y) \notin U_{m}$. Let us remark that $n(y)=\infty$ if $y \in \Lambda$. It is easy to see that

$$
y \in X_{n(y)} \mid X_{n(y)+1} \text { if } n(y)<\infty \text {. }
$$

If $z \in W$ then we define $\rho_{\mathscr{F}^{u}, n}(y, z)$ by means of formula (1.3) assuming that $n \in$ $[0, n(y)]$. Let $Y_{n}$ be a $\gamma_{n}$-neighbourhood of $X_{n}$. As $\bar{X}_{n} \subset X_{n-1}$ we get for small enough $\gamma_{n}$ that $\bar{Y}_{n} \subset X_{n-1}$. The following assertion is a consequence of proposition 2 and lemma 8. 
LEMMA 11. There exist continuous functions $\tilde{\kappa}(y, z), \tilde{\kappa}_{n}(y, z)(n=0,1,2, \ldots)$ defined for $y \in X, z \in \tilde{W}(y)$ such that

(1) $\tilde{\kappa}(y, z)=\rho_{\mathscr{g}^{u}}(y, z)$ for $y \in X \cap \Lambda$;

(2) $\tilde{\kappa}_{n}(y, z)=\rho_{\mathscr{F}^{\mu}, n}(y, z)$ for $y \in X_{n}$;

(3) $\tilde{\kappa}_{n}(y, z)=\tilde{\kappa}(y, z)$ for $y \in X \backslash Y_{n}$;

(4) the sequence $\left\{\tilde{\kappa}_{n}\right\}$ converges uniformly to function $\tilde{\kappa}$.

Assertion 4 of lemma 5 implies that for any integer $s>0$

$$
\bigcup_{y \in \bar{U}_{m} \backslash U_{m+1}} \tilde{W}(y) \cap \bar{U}_{m+s}=\varnothing .
$$

Let $V_{0}=U_{m+s}, V_{0}^{\prime} \subset V_{0}$ be a neighbourhood of $\Lambda, \Psi(x)$ be a non-negative density function (satisfying (1.5)), and $h$ be a continuous function with the support in $V_{0}^{\prime} \cap \Pi$.

LEMMA 12. For any $x \in V_{0}^{\prime} \backslash P_{n}$

$$
\Psi(x) h\left(f^{n}(x)\right)=0 .
$$

Proof. Let $x \in V_{0}^{\prime} \backslash P_{n}$. We assume that $\Psi(x) h\left(f^{n}(x)\right) \neq 0$. Then $f^{n}(x) \in \Pi$. On the other hand from $x \in V_{0}^{\prime}$ we get

$$
f^{n}(x) \in f^{n}\left(V_{0}^{\prime}\right) \subset f^{n}\left(U_{m+s}\right)=U_{m+n+s} .
$$

Therefore $f^{n}(x) \in U_{m+n+s} \cap \Pi$. It follows from (2.24) that the intersection $U_{m+n+s} \cap$ $\Pi$ is contained in $\Pi_{n}$. Therefore $x \in f^{-n}\left(\Pi_{n}\right)=P_{n}$. This contradiction proves our assertion.

We get, using lemmas 10 and 12 , that

$$
\begin{aligned}
& \int_{V_{0}^{\prime}} \Psi(x) h\left(f^{n}(x)\right) d \nu(x) \\
&=\int_{P_{n}} \Psi(x) h\left(f^{n}(x)\right) d \nu(x) \\
&=\int_{P_{n} / \eta_{n}} d \nu_{n}\left(y^{\prime}\right) \int_{C_{\eta_{n}}\left(y^{\prime}\right)} \Psi\left(z^{\prime}\right) \rho_{y^{\prime}}^{n}\left(z^{\prime}\right) h\left(f^{n}\left(z^{\prime}\right)\right) d \nu_{n, y^{\prime}}\left(z^{\prime}\right) \\
&=\int_{P_{n} / \eta_{n}} \Psi\left(y^{\prime}\right) d \nu_{n}\left(y^{\prime}\right) \int_{C_{\eta_{n}}\left(y^{\prime}\right)} \rho_{y^{\prime}}^{n}\left(z^{\prime}\right) h\left(f^{n}\left(z^{\prime}\right)\right) d \nu_{n, y^{\prime}}\left(z^{\prime}\right) \\
&+\int_{P_{n} / \eta_{n}} d \nu_{n}\left(y^{\prime}\right) \int_{C_{\eta_{n}}\left(y^{\prime}\right)}\left(\Psi\left(z^{\prime}\right)-\Psi\left(y^{\prime}\right)\right) \rho_{y^{\prime}}^{n}\left(z^{\prime}\right) h\left(f^{n}\left(z^{\prime}\right)\right) d \nu_{n, y^{\prime}}\left(z^{\prime}\right) \\
&= I_{1}^{(n)}+I_{2}^{(n)} .
\end{aligned}
$$

It follows from lemma 8 that

$$
\operatorname{diam} C_{\eta_{n}}\left(y^{\prime}\right) \leq K_{13}\left(\mu_{1}-\varepsilon\right)^{-n}
$$

where $K_{13}>0$ is a constant independent on $n$. Therefore the uniform continuity of the function $\Psi(x)$ implies that

$$
\left|\Psi\left(z^{\prime}\right)-\Psi\left(y^{\prime}\right)\right|=\varepsilon_{n} \rightarrow 0
$$

as $n \rightarrow \infty$ uniformly in $y^{\prime} \in P_{n} / \eta_{n}$ and $z^{\prime} \in C_{\eta_{n}}\left(y^{\prime}\right)$. Thus

$$
I_{2}^{(n)} \leq \varepsilon_{n} \max _{x \in V^{\prime}} h(x) \rightarrow 0 \quad \text { as } n \rightarrow \infty \text {. }
$$


Assume that $y=f^{n}\left(y^{\prime}\right), z=f^{n}\left(z^{\prime}\right)$. Then

$$
y \in \Pi_{n} / \xi=X_{n}, \quad z \in f^{n}\left(C_{\eta_{n}}\left(y^{\prime}\right)\right)=\tilde{W}(y) .
$$

We have

$$
d \nu_{n}\left(y^{\prime}\right)=\mathscr{J}_{X}^{(n)}(y) d \nu_{X}(y)
$$

where $\mathscr{J}_{X}^{(n)}$ is the Jacobian of the map $f^{n} \mid X$ at $y, \nu_{X}(y)$ is the measure on $X$, induced by Riemannian metric,

$$
d \nu_{n, y^{\prime}}\left(z^{\prime}\right)=\prod_{K=0}^{n-1} \mathscr{J}^{u}\left(f^{-K}(z)\right) d \nu_{y}(z)
$$

and $\nu_{y}(z)$ is the measure on $\tilde{W}(y)$, induced by Riemannian metric. We denote

$$
\begin{aligned}
\Psi_{n}(y) & =\Psi\left(f^{-n}(y)\right)=\Psi\left(y^{\prime}\right), \\
\tilde{\rho}_{y}^{(n)}(z) & =\rho_{f^{-n}(y)}^{(n)}\left(f^{-n}(z)\right)=\rho_{y^{\prime}}^{(n)}\left(z^{\prime}\right) .
\end{aligned}
$$

In view of lemma 11

$$
\begin{aligned}
I_{1}^{(n)}= & \int_{X_{n}} \Psi_{n}(y) \mathscr{J}_{X}^{(n)}(y) d \nu_{X}(y) \int_{\tilde{W}(y)} \tilde{\rho}_{y}^{(n)}(z) h(z) \prod_{K-n}^{n-1} \mathscr{J}^{u}\left(f^{-K}(z)\right) d \nu_{y}(z) \\
= & \int_{X} \Psi_{n}(y) \mathscr{J}_{X}^{(n)}(y) \chi_{X_{n}}(y) \prod_{K=0}^{n-1} \mathscr{J}^{u}\left(f^{-K}(y)\right) d \nu_{X}(y) \\
& \times \int_{\tilde{W}(y)} \tilde{\rho}_{y}^{(n)}(z) h(z) \tilde{\kappa}_{n}(y, z) d \nu_{y}(z) \\
= & \int_{X} \alpha_{n}(y) d \nu_{X}(y) \int_{\tilde{W}(y)} \tilde{\rho}_{y}^{(n)}(z) \tilde{\kappa}_{n}(y, z) \beta_{n}(y) h(z) d \nu_{y}(z)
\end{aligned}
$$

where

$$
\beta_{n}(y)=\left[\int_{\tilde{W}(y)} \tilde{\rho}_{y}^{(n)}(z) \tilde{\kappa}_{n}(y, z) d \nu_{y}(z)\right]^{-1}
$$

is the normalizing factor and

$$
\alpha_{n}(y)=\Psi_{n}(y) \mathscr{F}_{X}^{(n)}(y) \chi_{X_{n}}(y) \prod_{K=0}^{n-1} \mathscr{J}^{u}\left(f^{-K}(y)\right) \beta_{n}^{-1}(y) .
$$

Now the result follows from $(2.25),(2.23)$, lemma 11 , the continuity of the functions $\tilde{\kappa}_{n}(y, z), \tilde{\rho}_{y}^{(n)}(z), \beta_{n}(y)$, the definition of the limit measure $\mu\left(l\left(V_{0}^{\prime}, \Psi\right)\right)$ (cf. [5]) and the following lemma.

LEMMA 13. Let $\nu_{n}$ be a sequence of measures in $\Pi$, with the following properties:

(1) if $\left(\delta_{n}, \nu_{n}(y, z)\right)$ is the system of conditional measures for $\nu_{n}$ with respect to the partition $\xi$ so that $\delta_{n}(y)$ is the measure on $X$, and $\nu_{n}(y, z)$ is the measure on $\tilde{W}(y)$, then

$$
d \nu_{n}(y, z)=P_{n}(y, z) d \nu_{y}(z),
$$

where $P_{n}(y, z)$ is a continuous function on $\bar{\Pi}$;

(2) the sequence of functions $P_{n}(y, z)$ converges uniformly in $\bar{\Pi}$ to a continuous function $P(y, z)$ as $n \rightarrow \infty$;

(3) the sequence of measures $\mu_{n_{l}}=\left(1 / n_{l}\right) \sum_{k=0}^{n_{i}-1} \nu_{k}$ converges weakly in $\bar{\Pi}$ to a measure $\mu$, where $n_{l}$ is a subsequence of integers. 
Then the system of conditional measures for the measure $\mu$ in $\bar{\Pi}$ with respect to the partition $\xi$ has the form $(\delta(y), \nu(y, z))$, where $\delta(y)$ is a measure on $\bar{X}$, and $\nu(y, z)$ is the measure in $\tilde{W}(y)$ for which

$$
d \nu(y, z)=P(y, z) d \nu_{y}(z)
$$

Proof. We have for any continuous function $h$ in $\bar{\Pi}$ in view of condition 1 of the lemma that

$$
\begin{aligned}
\int_{\bar{\Pi}} h(w) d \mu_{n_{l}}(w) & =\frac{1}{n_{l}} \sum_{K=0}^{n_{l}-1} \int_{\bar{X}} d \delta_{K}(y) \int_{\tilde{W}(y)} h(y, z) P_{K}(y, z) d \nu_{y}(z) \\
& =\int_{\bar{X}} d \Psi_{n_{l}} \int_{\bar{W}(y)} h(y, z) P(y, z) d \nu_{y}(z)+\varepsilon_{n_{l}},
\end{aligned}
$$

where $\Psi_{n_{l}}=\left(1 / n_{l}\right) \sum_{K=0}^{n_{l^{-}-1}} \delta_{K}$ and

$$
\varepsilon_{n_{l}}=\frac{1}{n_{l}} \sum_{K=0}^{n_{l}-1} \int_{\bar{X}} d \delta_{K}(y) \int_{\tilde{W}(y)} h(y, z)\left(P_{K}(y, z)-P(y, z)\right) d \nu_{y}(z) .
$$

Supposing

$$
c=\max _{(y, z) \in \bar{\Pi}}|h(y, z)| \quad \text { and } \quad c_{K}=\max _{(y, z) \in \bar{\Pi}}\left|P_{K}(y, z)-P(y, z)\right|
$$

and taking into account condition 2 of the lemma we have

$$
\left|\varepsilon_{n_{l}}\right| \leq \frac{c}{n_{l}} \sum_{K=0}^{n_{l}-1} c_{K} \rightarrow 0 \quad \text { as } n_{l} \rightarrow \infty .
$$

We consider in $\bar{\Pi}$ the sequence of measures $\tilde{\mu}_{n_{l}}$, where

$$
\tilde{\mu}_{n_{l}}(A)=\int_{\tilde{X}} d \psi_{n_{l}} \int_{\tilde{W}(y)} \chi_{A}(y, z) P(y, z) d \nu_{y}(z)
$$

where $A \subset \Pi$ is a Borel set. It follows from what was said above that the measures $\tilde{\mu}_{n_{l}}$ converge weakly to the measure $\mu$. Thus the family $\left\{\Psi_{n_{t}}\right\}$ is weakly compact. Therefore we get using the compactness of $\bar{X}$ that measures the $\Psi_{n_{i}}\left(n_{l}^{\prime}\right.$ is a subsequence of $n_{l}$ ) converge weakly to a measure $\delta$ on $\bar{X}$. It means in view of the continuity of the function $P(y, z)$ that for any continuous function $h$ in $\Pi$

$$
\begin{aligned}
\int_{\bar{\Pi}} h(w) d \tilde{\mu}_{n_{i}}(w) & =\int_{\bar{X}} d \Psi_{n_{i}}(y) \int_{\dot{W}(y)} h(y, z) P(y, z) d \nu_{y}(z) \\
& \rightarrow \int_{\bar{X}} d \delta(y) \int_{\tilde{W}(y)} h(y, z) P(y, z) d \nu_{y}(z)
\end{aligned}
$$

as $n_{l}^{\prime} \rightarrow \infty$. On the other hand

$$
\int_{\bar{n}} h(w) d \tilde{\mu}_{n_{i}}(w) \rightarrow \int_{\bar{n}} h(w) d \mu(w)
$$

as $n_{l}^{\prime} \rightarrow \infty$. Now the result which we need follows from two last co-relations. Moreover the measures $\Psi_{n}$ converge weakly in $\bar{X}$ to the measure $\delta$. 
2.6. Proof of proposition 3. We have for any $n>0$ and $A \subset \Lambda$

$$
\begin{aligned}
\nu_{n}(A) & =\tilde{\nu}_{n}\left(A \cap U_{n}\right)=\tilde{\nu}_{n}\left(f^{n}\left(f^{-n}(A) \cap U_{0}\right)\right) \\
& =\int_{f^{-n}(A) \cap U_{0}} c_{n} \rho_{\mathcal{F}^{u}}\left(f^{n}(x), f^{n}(y)\right) \prod_{K=0}^{n-1} \mathscr{J}^{u}\left(f^{K}(y)\right) d \nu^{u}(y) \\
& =\int_{f^{-n}(A) \cap U_{0}} c_{n} \rho_{\mathcal{F}^{u}}\left(f^{n}(x), f^{n}(y)\right) \prod_{K=0}^{n-1} \mathscr{F}^{u}\left(f^{K}(y)\right)\left[\rho_{\mathcal{F}}{ }^{u}(x, y)\right]^{-1} d \tilde{\nu}_{0}(y) \\
& =\int_{f^{-n}(A) \cap U_{0}} c_{n} \prod_{K=0}^{n-1} \mathscr{J}^{u}\left(f^{K}(x)\right) d \tilde{\nu}_{0}(y)=\int_{f^{-n}(A) \cap U_{0}} d \tilde{\nu}_{0}(y) \\
& =\tilde{\nu}_{0}\left(f^{-n}(A) \cap U_{0}\right)=\nu_{0}\left(f^{-n}(A)\right) .
\end{aligned}
$$

2.7. Proof of theorem 4. Let $x$ be a density point for a limit measure $\mu, \Pi(x, W, r)$ be a $\Lambda$-rectangle at $x, \xi$ be a partition of $\Pi(x, W, r)$ into the sets $B^{u}(y, r), y \in W \cap \Lambda$. We identify $\bar{\Pi}(x, W, r) / \xi$ with $\bar{W} \cap \Lambda$ and denote

$$
\begin{gathered}
A_{n}=\left\{y \in \bar{W}: B^{u}(y, r) \cap U_{n} \neq \varnothing\right\}, \\
B_{n}=\left\{y \in \bar{W}: B^{u}(y, r) \cap \partial U_{n} \neq \varnothing\right\} \\
C_{n}=A_{n} \backslash B_{n}, \quad D_{n}=\bigcup_{y \in B_{n}} B^{u}(y, r), \\
Q_{n}=\left\{y \in W^{u}\left(f^{n}(x)\right): \rho_{f_{(x)}^{n}}^{u}\left(y, \partial U_{n}\right) \leq 2 r\right\},
\end{gathered}
$$

where $\rho_{x}^{u}$ is the distance in $W^{u}(x)$, induced by Riemannian metric. It is easy to see that

$$
B_{n} \subset A_{n}, \quad D_{n} \subset Q_{n}
$$

and $C_{n}$ consists of a finite number of elements. It follows from the definitions of measures $\tilde{\nu}_{n}$ and numbers $c_{n}$, assertion 3 of proposition 1 and assertion 2 of proposition 2 that

$$
\begin{aligned}
\tilde{\nu}_{n}\left(Q_{n}\right) & =c_{n} \int_{Q_{n}} \rho_{\mathscr{F}^{u}}\left(f^{n}(x), y\right) d \nu^{u}(y) \\
& =c_{n} \int_{f^{-n}\left(Q_{n}\right)} \rho_{\mathscr{F}^{u}}\left(f^{n}(x), f^{n}\left(y^{\prime}\right)\right) \prod_{K=0}^{n-1} \mathscr{F}^{u}\left(f^{K}\left(y^{\prime}\right)\right) d \nu^{u}\left(y^{\prime}\right) \\
& =\int_{f^{-n}\left(Q_{n}\right)} \rho_{\mathscr{F}^{u}}\left(x, y^{\prime}\right) d \nu^{u}\left(y^{\prime}\right) \leq K_{14} \nu\left(f^{-n}\left(Q_{n}\right)\right) \\
& \leq K_{15}\left(\mu_{1}-\varepsilon\right)^{--n} 2 r,
\end{aligned}
$$

where $K_{14}>0, K_{15}>0$ are constants. We denote by $\delta_{n}$ the measure on $W$, concentrated on $C_{n}$, whose value on the set $Z \subset C_{n}$ is equal to the average number of points in $Z$. Let $h$ be a continuous function on $\Lambda$ whose support belongs to $\Pi\left(x_{0}, W, r\right)$. Denoting by $c=\max _{y \in \Lambda}|h(y)|$ we have

$$
\begin{aligned}
\int_{\Lambda} h(y) d \nu_{n}(y) & =\int_{\Pi\left(x_{0}, W, r\right)} h(y) d \nu_{n}(y) \\
& =\sum_{y \in A_{n}} \int_{B^{u}(y, r) \cap U_{n}} h(z) d \tilde{\nu}_{n}(z) \\
& =\sum_{y \in C_{n}} \int_{B^{u}(y, r) i U_{n}} h(z) d \tilde{\nu}_{n}(z)+\sum_{y \in B_{n}} \int_{B^{u}(y, r) \cap U_{n}} h(z) d \tilde{\nu}_{n}(z) \\
& =I_{1}^{(n)}+I_{2}^{(n)} .
\end{aligned}
$$


It follows from (2.26) and (2.27) that

$$
I_{2}^{(n)} \leq c \nu\left(D_{n}\right) \leq c \nu\left(Q_{n}\right) \leq 2 c K_{15} r\left(\mu_{1}-\varepsilon\right)^{-n} .
$$

Then we have in view of the definition of the measure $\nu_{n}$ and assertion 3 of proposition 2 that

$$
\begin{aligned}
I_{1}^{(n)} & =c_{n} \sum_{y \in C_{n}} \rho_{\mathscr{F}^{u}}\left(f^{n}(x), y\right) \int_{B^{u \prime}(y, r)} h(z) \rho_{\mathscr{J}^{u}}(y, z) d \nu^{u}(z) \\
& =\int_{W} c_{n} \rho_{\mathscr{J}^{u}}\left(f^{n}(x), y\right) \beta(y) d \delta_{n}(y) \int_{B^{u}(y, z)} h(z) \frac{\rho_{\mathscr{J}^{u}}(y, z)}{\beta(y)} d \nu^{u}(z),
\end{aligned}
$$

where $\beta(y)=\int_{B^{u}(y, r)} \rho_{\mathscr{F}^{u}}(y, z) d \nu^{u}(z)$. Now the result follows from (2.28) and lemma 13.

2.8. Proof of theorem 5. It follows from results in [14] that for any $\varepsilon>0$ there exists a neighbourhood $U \subset \operatorname{Diff}^{(1+\alpha)}(U, M)$ of the diffeomorphism $f$ such that assertions 1-5 of the theorem hold but we know only that the set $\Lambda$ is partially hyperbolic. In order to prove that it is a partially hyperbolic attractor we need to check the condition A3. To do this it is sufficient to prove that

$$
W_{g}^{u}(x) \subset \Lambda_{g} \text {. }
$$

We suppose for $x \in \Lambda_{\mathrm{g}}$

$$
Z_{\mathrm{g}}^{u}(x)=\left\{y \in U_{0}: \rho\left(f^{n}(y), f^{n}\left(W_{\mathrm{g}}^{0}(x)\right)\right) \rightarrow 0 \text { as } n \rightarrow-\infty\right\} .
$$

One can show easily that

$$
Z_{\mathrm{g}}^{u}(x)=\bigcup_{y \in W_{\mathrm{g}}^{0}(x)} W_{\mathrm{g}}^{u}(x) .
$$

From (2.30), assertion 3 of proposition 1 and the conditions of the theorem we get $Z_{f}^{u}(x) \subset \Lambda$. Therefore using assertion 4 of the theorem and the definition of the sets $Z_{g}^{u}(x)$ we get

$$
Z_{g}^{u}(x)=h_{g}\left(Z_{f}^{u}(x)\right) \subset \Lambda_{g} .
$$

The last inclusion and (2.30) imply (2.29).

\section{Examples and applications}

3.1. The following assertion is a direct consequence of theorems 1 and 3 .

THEOREM 6. Let $f: M \rightarrow M$ be a partially hyperbolic $C^{1+\alpha}$-diffeomorphism of a smooth compact Riemannian manifold $M$ (cf. [3]), $\nu$ be a measure in $M$ which is absolutely continuous with respect to the Riemannian volume. Then any weak limit measure $\mu$ of the sequence $\mu_{n}=(1 / n) \sum_{K=0}^{n-1} f_{*}^{K} \nu$ is an f-invariant u-measure. Moreover, $h_{\mu}(f)>0$.

3.2. THEOREM 7. Let $\Lambda$ be a partially hyperbolic attractor of a $C^{1+\alpha}$-diffeomorphism $f: U \rightarrow M$. Then there exists a neighbourhood $V \subset U$ of $\Lambda$ and a continuous distribution $E$ in $T U$ such that

(1) $E(x)=E^{\prime \prime}(x)$ for any $x \in \Lambda$;

(2) $E(x)$ satisfies in $U$ Hölder's condition;

(3) $E(x)$ is smooth on set $U \backslash \Lambda$;

(4) $d f E(x)=E(f(x))$ for any $x \in U$. 
The proof follows from lemma 7. We can take as $E(x)$ the distribution $T_{x} W(x)$ where $x \in U_{m}$.

There exist, in general, infinitely many distributions with similar properties. They can be obtained by 'forward' iterations of smooth initial distributions given in the set $\bar{U}_{m} \mid U_{m+1}$ and selected in a special way.

The submanifolds $\exp _{x} W(x)$ are the natural candidates for local integrable manifolds for these distributions. However, it is unknown whether we can 'paste together' from them a global $f^{n}$-invariant $(n=1,2, \ldots)$ foliation in a neighbourhood of attractor $\Lambda$. It can be easily done only in the special case of co-dimension one attractors with the help of theorem 7 and the theorem of existence and uniqueness in the theory of ordinary differential equations.

THEOREM 8. Let $\Lambda$ be a partially hyperbolic attractor of a $C^{1+\alpha}$-diffeomorphism $f: U \rightarrow M$. Assume that $\Lambda$ has co-dimension one (i.e. $\operatorname{dim} E^{u}(x)=1$ ). Then there exists a neighbourhood $U$ of the set $\Lambda$ and a continuous $C^{1}$-foliation $W$ such that:

(1) $W(x)=W^{u}(x)$ for any $x \in \Lambda$;

(2) the foliation $W$ satisfies Hölder's condition in $U$;

(3) the foliation $W$ is smooth on set $U \backslash \Lambda$;

(4) $W(x) \subset \Lambda$ for any $x \in \Lambda$ and $W(x) \subset U \backslash \Lambda$ for any $x \in U \backslash \Lambda$;

(5) $f(W(x))=W(f(x)), x \in U$;

(6) the foliation $W$ is absolutely continuous with respect to the Riemannian volume.

3.3. In this and the next section we shall describe two constructions, leading to partially hyperbolic attractors. Let $\Lambda$ be a hyperbolic attractor of a $C^{1+\alpha}$ diffeomorphism $f: U \rightarrow M$ (it means that in addition to (1.1) $\lambda_{2}<1$ ), and $N$ be a smooth compact manifold. Suppose

$$
\tilde{U}=U \times N, \quad \tilde{\Lambda}=\Lambda \times N, \quad \tilde{M}=M \times N
$$

and consider the diffeomorphism $\tilde{f}: \tilde{U} \rightarrow \tilde{M}$, given by the formula

$$
\tilde{f}(x, y)=(f(x), y), x \in U, y \in N .
$$

It is easy to see that $\tilde{\Lambda}$ is a partially hyperbolic attractor for $f$, satisfying the conditions of theorem 5. Therefore this theorem and theorems 1 and 3 imply the following assertion.

THEOREM 9. For any $\varepsilon>0$ there exists a neighbourhood

$$
U \subset \operatorname{Diff}^{r}(\tilde{U}, \tilde{M})
$$

of the diffeomorphism $f$ such that

(1) any diffeomorphism $g \in U$ has a partially hyperbolic attractor $\Lambda_{\mathrm{g}}$;

(2) there exists a homeomorphism $h_{g}: \Lambda \rightarrow \Lambda_{g}$ such that

$$
\rho\left(h_{g}(x), x\right) \leq \varepsilon
$$

for any $x \in \Lambda_{\mathbf{g}}$

(3) the map $g \mid \Lambda_{g}$ has an invariant normalized Gibbs $u$-measure $\mu_{g}$, which can be constructed in the way described above and $h_{\mu_{g}}\left(g \mid \Lambda_{g}\right)>0$. 
3.4. Let $f^{t}$ and $g^{t}$ be two flows on manifolds $M$ and $N$, having hyperbolic attractors $\Lambda_{1}$ and $\Lambda_{2}$ respectively (for the definition of a hyperbolic attractor for a flow, see [16]). Let us fix $t$ and $s$ and consider the diffeomorphism $\phi_{t, s}: M \times N \rightarrow M \times N$ given by the formula

$$
\phi_{t, s}(x, y)=\left(f^{t}(x), g^{s}(y)\right), x \in M, y \in N .
$$

It is easy to see that set $\Lambda=\Lambda_{1} \times \Lambda_{2}$ is a partially hyperbolic attractor for $\phi_{t, s}$, where

$$
E^{s}=E_{f}^{s} \oplus E_{\mathrm{g}}^{s}, \quad E^{u}=E_{f}^{u} \oplus E_{\mathrm{g}}^{u}, \quad E^{0}=X_{f} \oplus X_{\mathrm{g}} .
$$

Here $E_{f}^{s}, E_{\mathrm{g}}^{s}$ and $E_{f}^{u}, E_{\mathrm{g}}^{u}$ are stable and unstable subspaces for the flows $f^{t} \mid \Lambda_{1}$ and $f^{t} \mid \Lambda_{2}$ respectively, and $X_{f}, X_{g}$ are one-dimensioned subspaces corresponding to vector fields of flows $f^{t}$ and $g^{t}$.

THEOREM 10. Theorems 1-5 hold for diffeomorphisms $\phi_{t, s}$ for any $t, s$.

The authors express their sincere gratitude to D. V. Anosov and M. I. Rabinovich for valuable discussions.

\section{REFERENCES}

[1] D. V. Anosov. On tangent fields of transversal foliation in ' $U$ '-systems. Mat. Zametky 2 No 5 (1967), 539-548.

[2] D. V. Anosov \& Ya. G. Sinai. Some smooth ergodic systems. Russ. Math. Surveys 22 No 5 (1967), 107-172.

[3] M. Brin \& Ya. Pesin. Partially hyperbolic dynamical systems. Izv. Acad. Nauk SSSR ser mat. No 1 (1974), 170-212.

[4] M. Brin. The estimation below of the entropy of smooth dynamical systems. Functional Anal. 8 No 3 (1974), 71-72.

[5] Ya. G. Sinai. Lectures on Ergodic Theory. Erevan State Univ. Press: Erevan, 1973.

[6] Ya. G. Sinai. Dynamical systems with Lebesgue spectrum II. Izv. Acad. Nauk SSSR 30 No 1 (1968), 15-68.

[7] Ya. B. Pesin. Characteristic Lyapunov exponents and smooth ergodic theory. Russ. Math. Surveys. 32 No 4 (1977), 55-114.

[8] Ya. B. Pesin. Description of $\pi$-partition for diffeomorphism with invariant measure. Mat. Zametky 21 No 6 (1977), 29-44

[9] Ya. B. Pesin. Families of invariant manifolds for dynamical systems with non-zero characteristic Lyapunov exponents. Izv. Acad. Nauk SSSR 40 No 6 (1976), 1332-1379.

[10] G. A. Margulis. On some problems in the theory of $U$-systems. Dissertation, Moscow State Univ. 1970.

[11] J. Mather. Characterization of Anosov diffeomorphisms. Indag. Math. 30 No 5 (1968), 479-483.

[12] A. Katok. Local properties of hyperbolic sets. Appendix to the Russian translation of the book by Z. Nitecki. Introduction to Differential Dynamics. Moscow: Mir Press, 1975.

[13] A. Katok. Lyapunov exponents, entropy and periodic orbits for diffeomorphisms. Publ. Math. 51 (1980), 137-173.

[14] M. Hirsch, P. Pugh \& M. Shub. Invariant manifolds. Bull. Amer. Math. Soc. 76 (1970), 1015-1019.

[15] R. Bowen. Equilibrium states and the ergodic theory of Anosov diffeomorphisms. Lecture Notes, No. 470,1975 , p. 108.

[16] R. Bowen \& D. Ruelle. The ergodic theory of axiom A flows. Invent. Math. 29 (1975), 181-202. 